\title{
Supporting Information Theory of microphase segregation in ABA triblock comb-shaped copolymers: lamellar mesophase
}

\author{
Ivan V.Mikhailov ${ }^{1}$, Frans A.M. Leermakers ${ }^{2}$, Anatoly A. Darinskii ${ }^{1}$, \\ Ekaterina B. Zhulina ${ }^{1}$, Oleg V. Borisov ${ }^{1,3}$ \\ ${ }^{1}$ Institute of Macromolecular Compounds \\ of the Russian Academy of Sciences, \\ St.Petersburg, Russia, \\ ${ }^{2}$ Physical Chemistry and Soft Matter, Wageningen University, \\ 6703 NB Wageningen, The Netherlands, \\ ${ }^{3}$ Institut des Sciences Analytiques et de Physico-Chimie pour \\ l'Environnement et les Matériaux, UMR 5254 CNRS UPPA, \\ Pau, France
}

April 5, 2021

\section{SF-SCF method}

The numerical SF-SCF method as SS-SCF method is based on the meanfield approximation but it is free from the constraints imposed by the strong stretching approximation. This approach is used discrete lattice representation of the space. The space is taken to be composed of lattice sites with characteristic length $a$ and the volume per cell fixed at $a^{3}$. The lattice sites are organized in lattice layers, which are located parallel to the grafting surface and are numbered as $z=0, \cdots, D / a+1$. The layers $z=0$ and $z=D / a+1$ correspond to the grafting surfaces, in the layers $z=1$ and $z=D / a$ the end segments of backbones are pinned. The volume fractions $\varphi$ of monomer units and the exchange chemical potential $u$ remain constant within the same layer and vary only in the $z$-direction. 
The backbone and side chains of the grafted bottle-brushes are modeled as freely-jointed chains in an external effective field $u(z)$ induced by the inter- and intramolecular interactions. The correlations of longer range are ignored. The overlap of two segments on a lattice site can be allowed, but it is prevented via the incompressibility condition

$$
\varphi_{l p}^{l e f t}(z)+\varphi_{b r}(z)+\varphi_{l p}^{\text {right }}(z)=1, \quad \forall z
$$

where subscript "br" indicates "bridge" macromolecules and "lp" indicates "loops" (left and right respectively).

In the case of the lamellar mesophase composed by bottle-brushes, the free energy per one macromolecule in the lattice SCF approximation is defined as the negative logarithm of the partition function of the bottle-brushe molecules on a cubic lattice in the field of an effective chemical potential $(-\ln Q)$ minus the work of this field $\sum_{z} u(z) \varphi(z)$ :

$F_{e l}=-X \ln Q_{b r}[u(z)]-(1-X) \ln Q_{l p}[u(z)]-\frac{1}{\sigma} \sum_{z=0}^{z=D} u(z)\left[\varphi_{b r}(z)+\varphi_{l p}(z)\right] \Delta z$,

where $\ln Q[u(z)] \equiv \ln Q[u(z)]-\ln Q[0]$ is the logarithm of the partition function of the bottle-brushe molecules on a cubic lattice (for "bridges" and "loops" correspondently) in the field of an effective chemical potential $u(z)$. The last term is the work of this effective potential field.

The chains in lamellar morphology are not considered as being identical. In our model, the chains are initially divided into "bridges" and "loops". The "bridges" are strictly grafted at the end segments into two opposite walls of the lamellar phase. The "loops" are grafted with both ends to one of the walls of the lamellar phase. This difference is specified in the model through the initial conditions for the forward and back propagators (see step 2 in section Algorithm description). Thus, the partition functions for bridges and loops are calculated differently. The free energy of elastic tension (equation 2) was calculated at a strictly specified fraction $X$ of "bridges". For each value of $X$, a separate simulation was carried out. The equilibrium fraction of "bridges" in the lamellar phase was determined from the minimum free energy of elastic tension plus the free energy of mixing of "bridges" and "loops" from the large data set of numerical simulations of the same polymer system at different value of $X$.

Calculation of the volume fraction profiles $\left(\varphi_{b r}(z), \varphi_{l p}(z)\right)$ and the potential profile $(u(z))$ corresponding to the minimimum of the free energy under 
the incompressibility condition is achieved by optimization of the functional:

$$
\mathcal{F}=F_{e l}\left\{\varphi_{l p}, \varphi_{b r}, u(z)\right\}+\sum_{z=0}^{z=D} \alpha(z)\left[\varphi_{b r}(z)+\varphi_{l p}(z)-1\right] \Delta z,
$$

where $\alpha(z)$ is Lagrange field (set of Lagrange multipliers).

Minimization of $\mathcal{F}$ with respect to the volume fractions $\varphi(z)$ allows to calculate the potential fields $u(z)$ :

$$
\frac{\delta \mathcal{F}}{\delta \varphi(z)}=0 \quad \Rightarrow \quad u(z) \leftarrow \alpha(z)
$$

Minimization of $\mathcal{F}$ with respect to the potential fields $u(z)$ provides a way to calculate the volume fraction distributions $\varphi(z)$ :

$$
\frac{\delta \mathcal{F}}{\delta u(z)}=0 \Rightarrow \varphi(z) \leftarrow \frac{\delta(-\ln Q)}{\delta u(z)}
$$

The last expression can be represented as a composition of propagator matrices (the composition law). See below for details.

The Lagrange field $\alpha(z)$ corresponding to the minimum of functional $\mathcal{F}$ (eq. 3) is calculated during the iterative procedure of gradient descent:

$$
\alpha(z) \leftarrow \alpha+\eta \frac{\delta \mathcal{F}}{\delta \alpha(z)}=\alpha(z)+\eta\left[\varphi_{b r}(z)+\varphi_{l p}(z)-1\right],
$$

where $\eta<1 / 2$ is the convergence step size.

\subsubsection{Algorithm description}

Step 0. Define an arbitrary Lagrange field:

$$
\alpha(z):=0
$$

Step 1. Calculate the Boltzmann statistical weights (probability to find any monomer unit in a given $z$-th layer):

$$
W_{B}(z):=\exp \{-\alpha(z)\}
$$

Step 2. Set up the initial conditions of propagators for each type of macromolecules ("bridges" and "loops").

Back propagator for side chains has following initial guess:

$$
G_{a r m, b}(z, s=n+1)=W_{B}(z)
$$


This means that the end groups numbered $s=n+1$ are free, i.e. the propagation of the ends of the side chains can start from each layer.

The forward and back propagators of the backbone in the case of a "bridge" must start the walk in the first layer $z=1$ and in the last layers $z=D$, respectively:

$$
G_{\text {main }, f}^{\text {bridge }}(z=1, s=1)=W_{B}(1) \quad G_{\text {main }, b}^{\text {bridge }}\left(z=D, s=N_{b}\right)=W_{B}(D)
$$

For the "left" and "right loops", the walking should start and finish in the same layer:

$$
\begin{gathered}
G_{\text {main }, f}^{\text {left }}(z=1, s=1)=W_{B}(1) \quad G_{\text {main }, b}^{\text {left }}\left(z=1, s=N_{b}\right)=W_{B}(1) \\
G_{\text {main }, f}^{\text {right }}(z=1, s=1)=W_{B}(D) \quad G_{\text {main }, b}^{\text {right }}\left(z=D, s=N_{b}\right)=W_{B}(D)
\end{gathered}
$$

Step 3. "Fill in" all the matrices - propagators:

$$
\begin{aligned}
& G_{f}(s, z):=W_{B}(z)\left[\frac{1}{6} G_{f}(s-1, z-1)+\frac{4}{6} G_{f}(s-1, z)+\frac{1}{6} G_{f}(s-1, z+1)\right] \\
& G_{b}(s, z):=W_{B}(z)\left[\frac{1}{6} G_{b}(s+1, z-1)+\frac{4}{6} G_{b}(s+1, z)+\frac{1}{6} G_{b}(s+1, z+1)\right]
\end{aligned}
$$

If the segment number $s$ corresponds to a branching point, the probability of its distribution is calculated as the probability of meeting two Markov chains at walking along the backbone and at walking along the side chain from the free end to the branching point:

$$
\begin{aligned}
G_{f}(s, z) & :=G_{f}(s, z) \cdot G_{a r m, b}(z, s=1) / W_{B}(z) \\
G_{b}(s, z) & :=G_{b}(s, z) \cdot G_{a r m, b}(z, s=1) / W_{B}(z)
\end{aligned}
$$

Step 4. "Fill in" propagators for forward walking along side chains from the branching points to the free ends:

$$
\begin{aligned}
& G_{\text {arm }, f}(s=1, z, k):=G_{\text {main }, f}(s=k m+1, z) \cdot G_{\text {main }, b}(s=k m+1, z) / W_{B}(z) \\
& G_{\text {arm }, f}(s, z, k):=\frac{W_{B}(z)}{6} \times \\
& \times\left[G_{\text {arm }, f}(s-1, z-1, k)+4 G_{\text {arm }, f}(s-1, z, k)+G_{a r m, f}(s-1, z+1, k)\right]
\end{aligned}
$$

where $k$ is a number of side chain. 
Step 5. Calculate the distribution over the layers of the auxiliary quantity $q(z)$ :

$$
\begin{gathered}
q(z)=\frac{1}{W_{B}(z)} \times \\
\times\left[\sum_{s=1}^{s=N b} G_{\text {main }, f}(z, s) G_{\text {main }, b}(z, s)+\sum_{k=1}^{k=N b / m-1} \sum_{s=1}^{s=n} G_{a r m, f}(z, s, k) G_{a r m, b}(z, s)\right]
\end{gathered}
$$

Step 6. According to the composition law, calculate the volume fraction distributions for "bridges" and "loops" ("left" and "right"):

$$
\begin{gathered}
\varphi_{b r}(z)=\frac{q_{b r}(z)}{\sum_{z=1}^{z=D} q_{b r}(z)} \sigma X \\
\varphi_{l p}^{(l e f t)}(z)=\frac{q_{l p}^{(l e f t)}(z)}{\sum_{z=1}^{z=D} q_{l p}^{(l e f t)}(z)} \sigma \frac{(1-X)}{2} ; \quad \varphi_{l p}^{(\text {right })}(z)=\frac{q_{l p}^{(\text {right })}(z)}{\sum_{z=1}^{z=D} q_{l p}^{(\text {right })}(z)} \sigma \frac{(1-X)}{2}
\end{gathered}
$$

Step 7. Calculate a new Lagrange field:

$$
\alpha(z):=\alpha(z)+\eta\left[\varphi_{b r}(z)+\varphi_{l p}^{(l e f t)}(z)+\varphi_{l p}^{(r i g h t)}(z)-1\right]
$$

Step 8. Find the deviation from the incompressibility condition:

$$
\epsilon=\sqrt{\sum_{z=1}^{z=D}\left[\varphi_{b r}(z)+\varphi_{l p}^{(l e f t)}(z)+\varphi_{l p}^{(r i g h t)}(z)-1\right]^{2}}
$$

If the deviation is higher than the specified value $\left(\epsilon>10^{-8}\right)$, return to Step 1. Otherwise, exit from loop.

\subsection{Calculation of free energy}

After exiting the program cycle described above, it is calculated the free energy per one chain grafted to one of the walls:

$$
\begin{array}{r}
F=F_{e l}+F_{m i x}=-X \ln Q_{b r}-(1-X) \ln Q_{l p} \\
-\frac{N}{D} \sum_{z=0}^{z=D} \alpha(z)\left[\varphi_{b r}(z)+\varphi_{l p}^{(l e f t)}(z)+\varphi_{l p}^{(\text {rigth })}(z)\right] \Delta z \\
+X \ln X+(1-X) \ln (1-X),
\end{array}
$$


where the corresponding partition functions $Q_{b r}$ and $Q_{l p}$ are calculated by the equations:

$$
\begin{array}{r}
Q_{b r}=\frac{\sum_{z=1}^{z=D} q_{b r}(z)}{X N \sigma}=\frac{a}{X D} \sum_{z=1}^{z=D} q_{b r}(z) \\
Q_{l p}=\frac{a}{(1-X) D} \sum_{z=1}^{z=D} q_{l p}^{(l e f t)}(z)=\frac{a}{(1-X) D} \sum_{z=1}^{z=D} q_{l p}^{(\text {right })}(z)
\end{array}
$$

where $q_{b r}(z)$ and $q_{l p}(z)$ are calculated at help of equation using in step 5 of previous section

\section{Elastic free energies $F_{b b}$ and $F_{s c}$ for comblike polymer in planar brush}

Transformation of loops in equivalent subchains (loops cut in two equal halves at the middle point) leads to insignificant change in the molecular potential $U(z)$ in the dry brush (see Figure S1). Below we calculate the elastic free energy of comb-shaped subchains that model loops in the analytical SS-SCF theory.

\subsection{Elastic free energy $F_{b b}$ of backbone}

Distribution of elastic tension in the backbone of comb-shaped polymer (Figure 5 in the main text) prompts that the contribution of the side chain in the elastic free energy of comblike polymer is small compared to that of backbone. Using the parabolic potential framework, we estimate in this Appendix the elastic free energy contributions due to the backbone and side chains for a comb-shaped macromolecule with fixed position $z_{P}$ of its last branching unit.

Similarity between trajectories of the backbone of a comblike polymer and a linear chain with DP $M$ in planar brushes allows us to approximate the relative position $z_{i} / z_{P}$ of $i$-th branching unit as

$$
\frac{z_{i}}{z_{P}} \approx \sin \left(\frac{\pi i}{2 P}\right)
$$

and implement the stretching function $E_{b b}$ of the backbone as

$$
E_{b b}\left(z_{P}, z\right)=\frac{\pi}{2 M} \sqrt{z_{P}^{2}-z^{2}}
$$




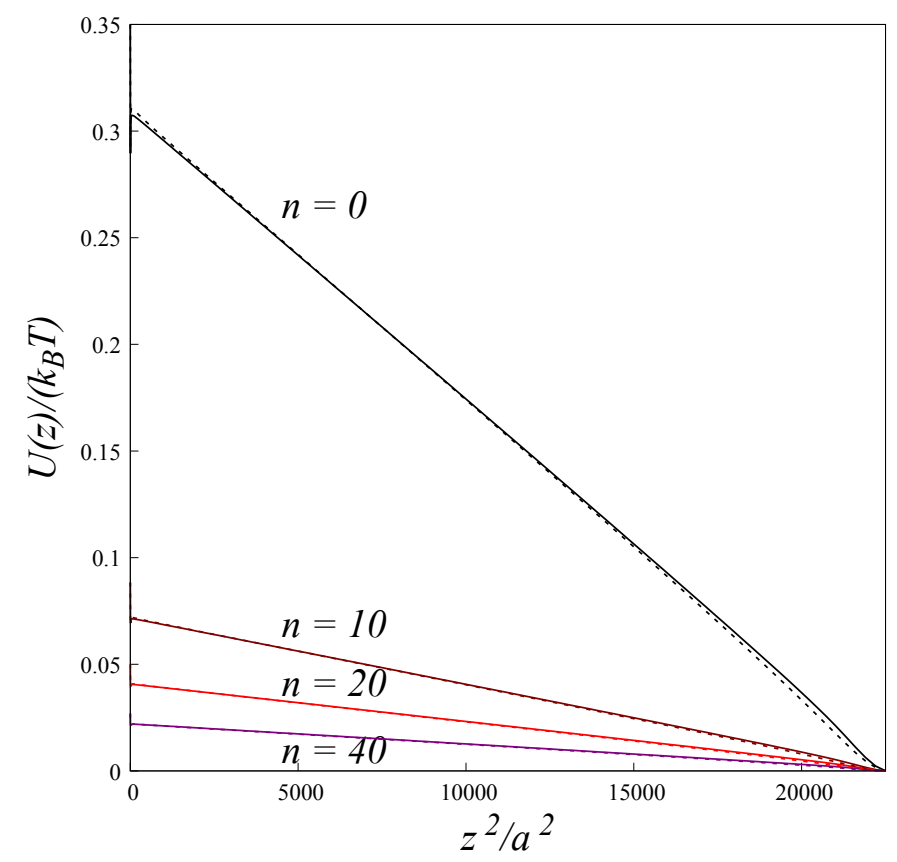

Figure S1: Numerically calculated SF-SCF molecular potential $U(z) /\left(k_{B} T\right)$ in solvent-free brush of loops (solid lines) and equivalent subchains (dashed lines) as a function of $z^{2} / a^{2}$ for $m=3$, fixed $2 D / a=300$ and $2 M=1050$, and varying $\mathrm{n}$. Transforming loops into subchains leads to a quite insignificant difference between two potentials. 
In the framework of SS-SCF approach, the elastic free energy of the backbone is then formulated as

$$
\frac{F_{b b}}{k_{B} T}=\frac{3}{2 l b} \int_{0}^{z_{P}} E_{b b}\left(z_{P}, z\right) d z=\frac{3 \pi^{2}}{16 l b} \frac{z_{P}^{2}}{M}
$$

\subsection{Elastic free energy $F_{s c}$ of side chains}

$P=M / m$ side chains are exposed to the molecular potential specified by eq 2 in the main text. The stretching function $E_{s c, i}$ of the side chain with DP $n$ originating from branching unit $i(i=1,2, \ldots P)$ is given by

$$
E_{s c, i}\left(z_{i}, z\right)=\kappa \sqrt{\frac{z_{i}^{2}}{\cos ^{2}(\kappa n)}-z^{2}}
$$

to give

$$
\begin{gathered}
\frac{F_{s c, i}}{k_{B} T}=\frac{3}{2 l b} \int_{z_{i}}^{z_{i} / \cos (\kappa n)} E_{s c, i}\left(z_{i}, z\right) d z=\frac{3}{4 l b} \kappa z_{i}^{2}\left[(\kappa n)\left(1+\tan ^{2}(\kappa n)\right)-\tan (\kappa n)\right] \approx \\
\approx \frac{z_{i}^{2}}{2 l b n}(\kappa n)^{4}, \quad \kappa n \ll 1
\end{gathered}
$$

The total elastic free energy $F_{s c}$ due to side chains yields

$$
\frac{F_{s c}}{k_{B} T}=q \sum_{1}^{P} \frac{F_{s c, i}}{k_{B} T}=\frac{z_{P}^{2}}{2 l b n}(\kappa n)^{4} q \sum_{i=1}^{i=P} \frac{z_{i}^{2}}{z_{P}^{2}} \approx \frac{z_{P}^{2}}{2 l b n}(\kappa n)^{4} q \sum_{i=1}^{i=P} \sin ^{2} \frac{\pi i}{2 P}
$$

By substituting summation by integration,

$$
\sum_{i=1}^{i=P} \sin ^{2} \frac{\pi i}{2 P} \approx \int_{0}^{P} \sin ^{2} \frac{\pi i}{2 P} d i=\frac{P}{2}
$$

one finds

$$
\frac{F_{s c}}{k_{B} T} \approx \frac{q}{4 l b} \frac{z_{P}^{2}}{u M} P^{2}(\kappa n)^{4}
$$

By substituting

$$
\kappa n=\frac{\pi}{2} \frac{\eta n}{N}=\frac{\pi}{2} \frac{\eta u}{P(1+q u)},
$$

implementing eq 4 from the main text,

$$
\eta=\left\{\begin{array}{cl}
q P, & P \ll(u / q)^{1 / 2} \\
(q u)^{1 / 2}, & P \gg(u / q)^{1 / 2}
\end{array}\right.
$$


and using

$$
\eta=(q u)^{1 / 2}, P \gg(u / q)^{1 / 2},
$$

the elastic free energy of side chains with $u \gg 1$ is finally evaluated as

$$
\frac{F_{s c}\left(z_{P}\right)}{k_{B} T} \approx \frac{\pi^{4}}{64 l b} \frac{z_{P}^{2}}{M} \frac{u}{q P^{2}}
$$

The ratio of the backbone and side chain contributions,

$$
\frac{F_{s c}\left(z_{P}\right)}{F_{b b}\left(z_{P}\right)} \approx \frac{\pi^{2}}{12} \frac{u}{q P^{2}}
$$

indicates that for barbwire $(q>1)$ macromolecules with number of repeats $P \gg(u / q)^{1 / 2}$, the elastic free energy of side chains is negligible compared with the elastic free energy of the backbone.

Therefore the average elastic free energy of the backbones, $\int_{0}^{D} g_{\text {comb }}\left(z_{P}\right) F_{b b}\left(z_{P}\right) d z_{P}$, in the brush of comblike polymers with distribution $g\left(z_{P}\right)$ of the free ends will also dominate over the average elastic free energy, $\int_{0}^{D} g_{c o m b}\left(z_{P}\right) F_{s c}\left(z_{P}\right) d z_{P}$, of the side chains. By taking advantage of the similarity between the distribution functions of the end-points of backbones, $g_{c o m b}\left(z_{P}\right)$, and of the free ends of linear chains in solvent-free brushes,

$$
g\left(z_{\text {end }}\right)=\frac{z}{D \sqrt{D^{2}-z^{2}}}
$$

one finds the average elastic free energy of comblike polymer per molecule,

$$
\begin{aligned}
\frac{F_{e l, c o m b}}{k_{B} T}= & \int_{0}^{D} g_{c o m b}\left(z_{P}\right)\left[\frac{F_{b b}\left(z_{P}\right)}{k_{B} T}+\frac{F_{s c}\left(z_{P}\right)}{k_{B} T}\right] d z_{P} \approx \\
& \int_{0}^{D} g_{c o m b}\left(z_{P}\right) \frac{F_{b b}\left(z_{P}\right)}{k_{B} T} d z_{P} \approx \\
& \frac{3 \pi^{2}}{16 l b M} \int_{0}^{D} \frac{z_{P}^{3} d z_{P}}{D \sqrt{D^{2}-z^{2}}}=\frac{\pi^{2} D^{2}}{8 l b M}
\end{aligned}
$$

\section{Solvent-free brush of loops with single side chain. SF-SCF model.}

Consider dry brushes of $Y$-shaped polymers (starlike macromolecule with stem DP $m$, and two branches with DPs $n$ and $m$ (see Figure S2). The branch with DP $m$ can form either loop or bridge. In Figures S3-S6 we 

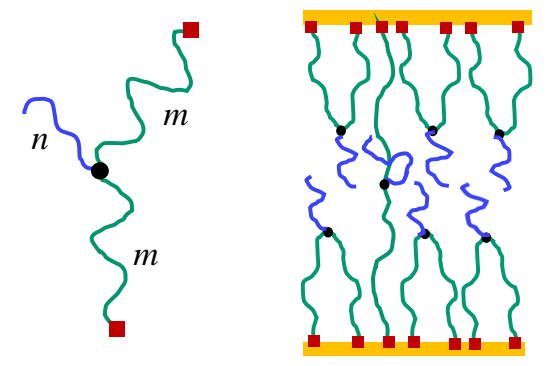

Figure S2: Schematic of Y-shaped polymer that makes either loop or bridge in two apposing dry brushes.

present the properties of apposing dry planar brushes formed by such polymers and demonstrate how an increase in $n$ affects the self-consistent molecular potential $U(z)$ (Figure S3), the elastic free energy $F$ per molecule and the equilibrium fraction $X_{m}$ of bridges (Figure $\mathrm{S} 4$ ), the volume fraction profile $\varphi_{M}(z)$ of the backbone monomer units (Figure S5), distribution $g_{m p}(z)$ of the middle monomer inits of in brushes of loops, and free end distribution $g(z)$ of equivalent subchains, i.e., loops cut in two equal parts (Figure S6). 
(a)

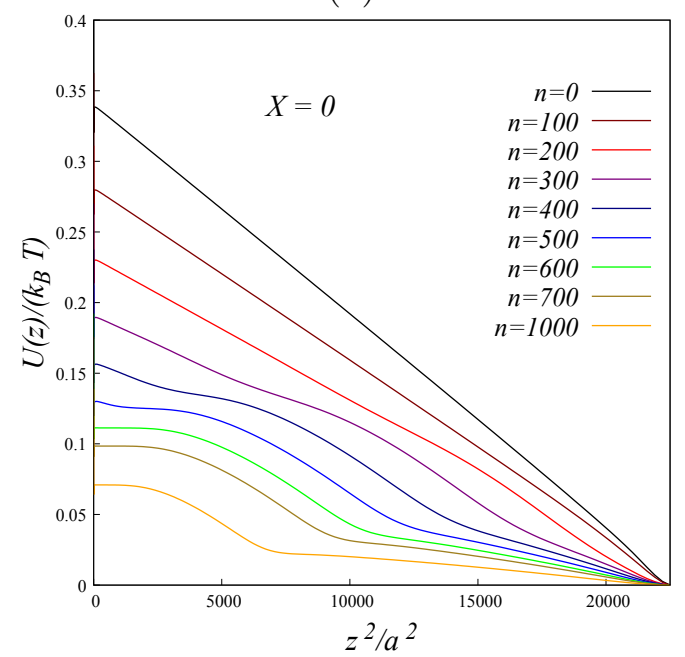

(b)

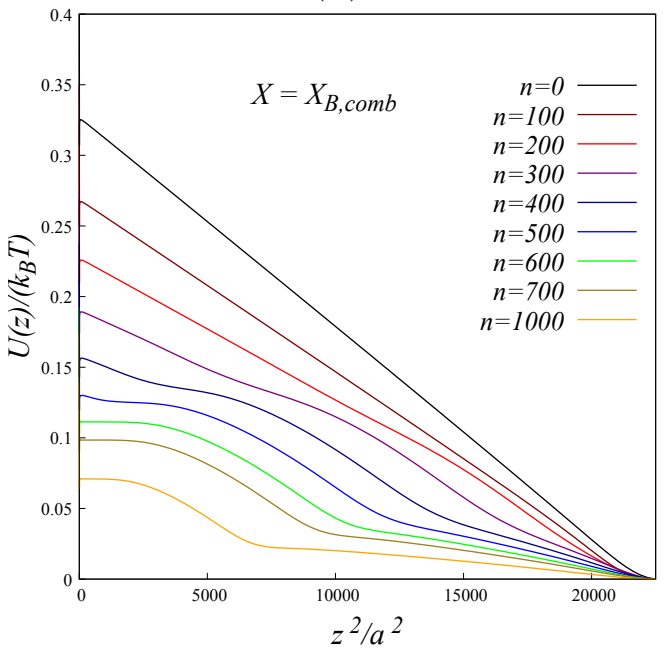

Figure S3: Dimensionless molecular potential $U(z) /\left(k_{B} T\right)$ as a function of $(z / a)^{2}$ in brushes of loops (a), and loops and bridges (b). $2 M=1001$, $2 D / a=300, m=500, n$ varies from 0 to 1000 .

(a)

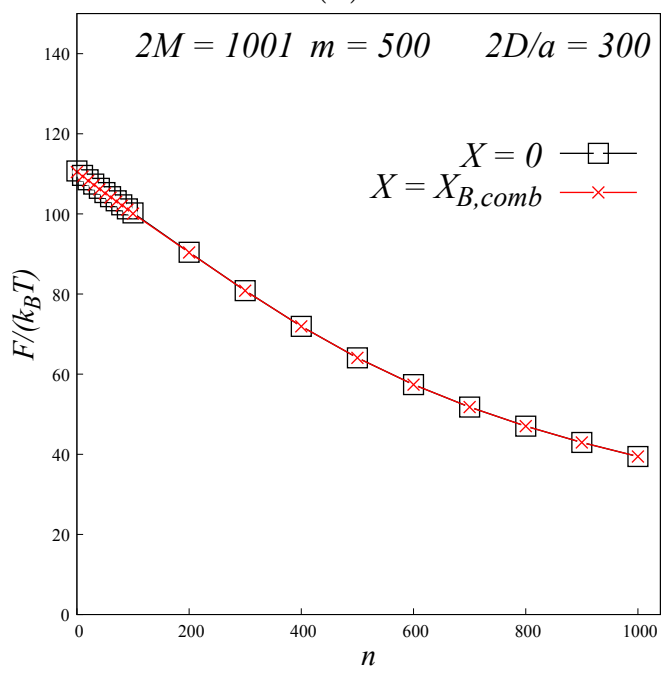

(b)

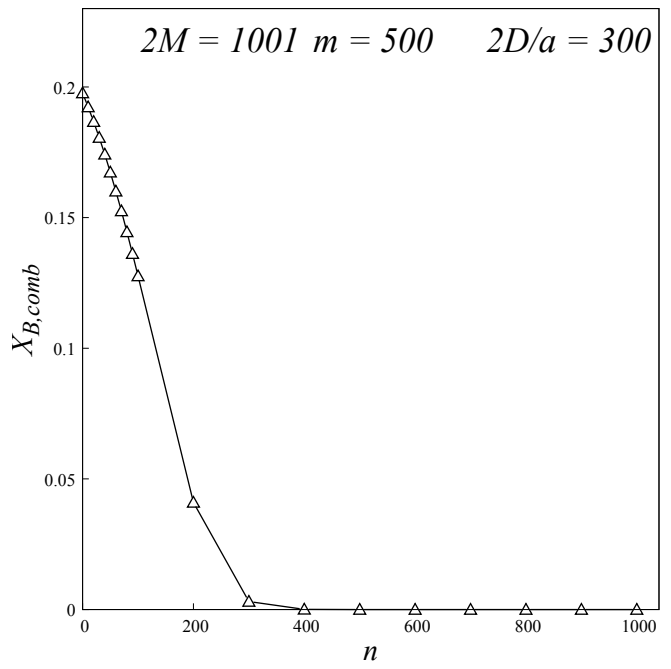

Figure S4: Free energy per chain (a) and equilibrium fraction of bridges (b) as a function of DP $n$. 
(a)

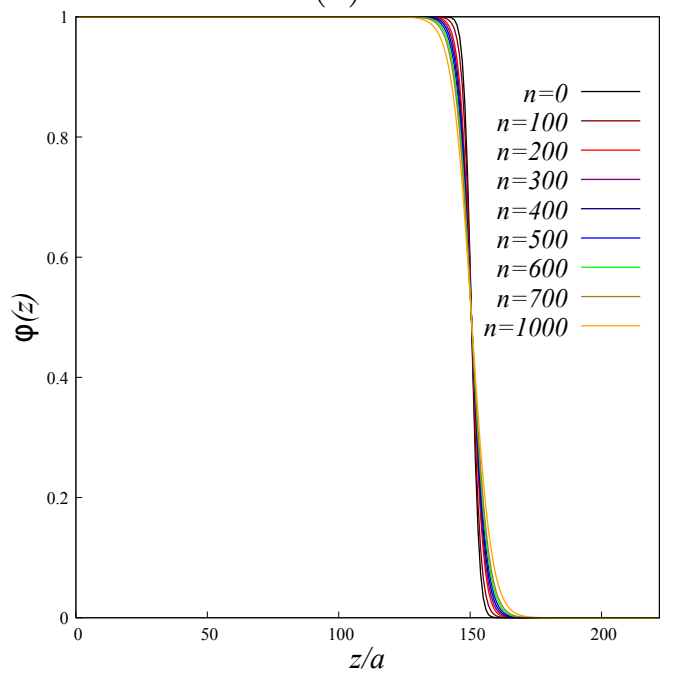

(b)

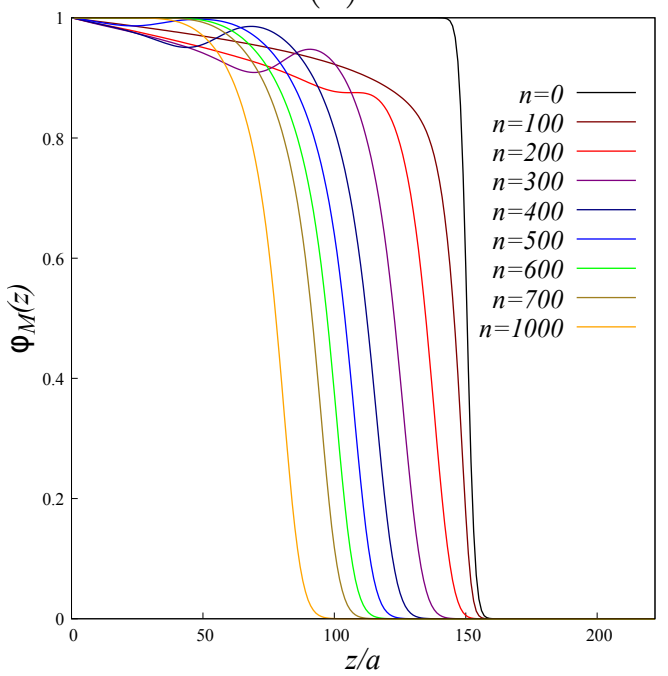

Figure S5: Volume fraction $\varphi(z)$ of all monomer units (a) and of backbone monomer units, $\varphi_{M}(z)$ (b) as a function of distance $z / a$ from the grafting surface.

(a)

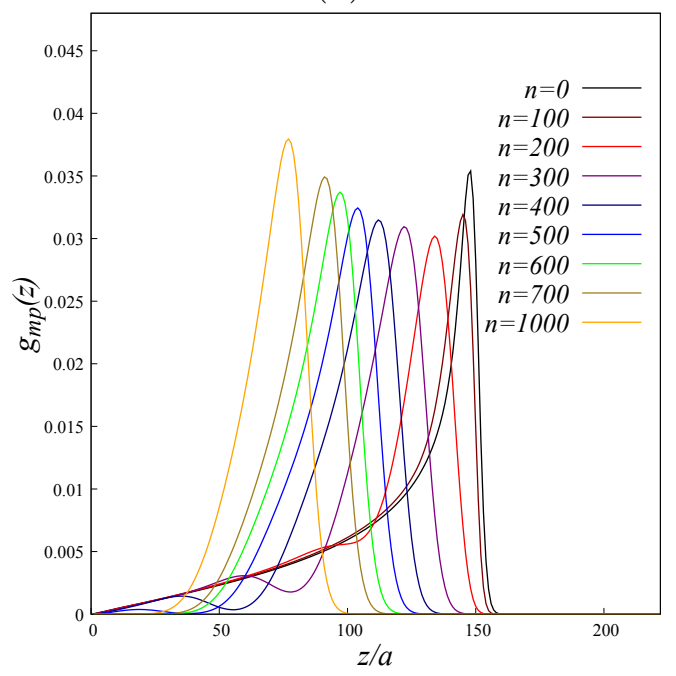

(b)

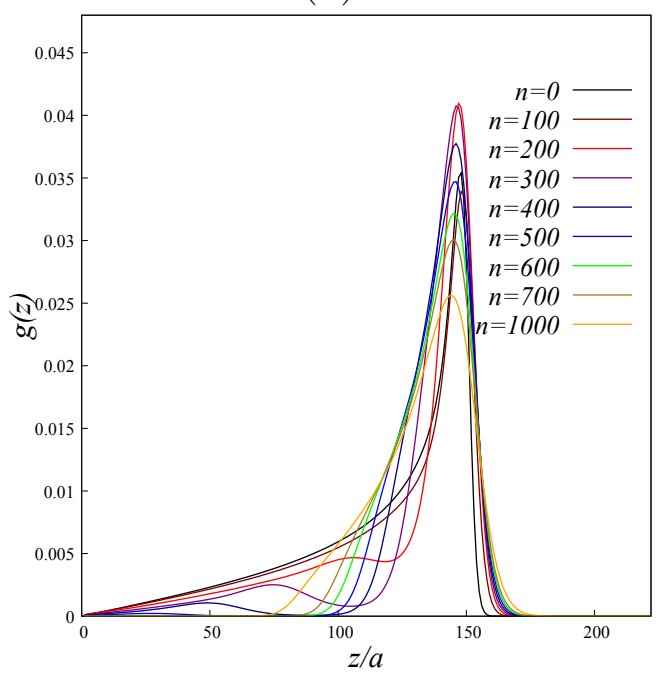

Figure S6: Volume fractions, $g_{m p}(z)$, of loop median monomer unit (a), and of end-points, $g(z)$, (b) as a function of distance $z / a$ from the grafting surface. 